OPEN

SUBJECT AREAS:

PHYSICAL CHEMISTRY

NANOSCALE DEVICES

TECHNIQUES AND INSTRUMENTATION

OTHER NANOTECHNOLOGY

Received

4 February 2013

Accepted

28 May 2013

Published

17 June 2013

Correspondence and requests for materials should be addressed to

L.S. (ling.sun@basf.

com)

\title{
Conductivity of individual particles measured by a microscopic four-point-probe method
}

\author{
Ling Sun', Jianjun Wang ${ }^{2}$ \& Elmar Bonaccurso 1,3
}

\begin{abstract}
${ }^{1}$ Max Planck Institute for Polymer Research, Ackermannweg 10, 55118 Mainz, Germany, ${ }^{2}$ Institute of Chemistry, Chinese Academy of Sciences, Zhongguancun North First Street 2, 100190 Beijing, PR China, ${ }^{3}$ Center of Smart Interfaces, Technical University of Darmstadt, Petersenstr. 32, 64287 Darmstadt, Germany.
\end{abstract}

We introduce a technique for measuring the conductivity of individual hybrid metal, semiconducting core-shell and full-metal conducting particles by a microscopic four-point probe $(\mu-4 \mathrm{PP})$ method. The four-point probe geometry allows for minimizing contact resistances between electrodes and particles. By using a focused ion beam we fabricate platinum nanoleads between four microelectrodes on a silicon chip and an individual particle, and determine the particle's conductivity via sensitive current and voltage measurements. Up to sixteen particles can be taken up by each chip, which allows for multiple conductivity measurements by simply multiplexing the electric contacts connected to a multimeter. Although, for demonstration, we used full Au (conducting) and Ag-coated latex particles (semiconducting) of a few micrometers in diameter, the method can be applied to other types of conducting or semiconducting particles of different diameters.

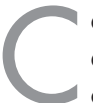

onjugated polymers are considered a most promising candidate in applications of biosensors and organic electronics due to their mechanical flexibility, processability, chemical versatility, and low $\cos ^{1,2}$. Most conjugated polymers are insoluble and infusible, which makes them difficult to be processed through solutions. However, after substitution with flexible side chains (e.g. long alkyl or alkoxy side chains) these polymers can also be processed, e.g. spin-coated or ink-jetted, from solution ${ }^{3,4}$. Another approach is to use latex particles as carriers of the conjugated polymers. Among others, we also showed this in previous works ${ }^{5,6}$ preparing core-shell particles consisting of a polystyrene core and a thin polypyrrole/polystyrenesulfonate (PPy/PSS) shell. One important question arising for such systems is how to measure the conductivity of individual polymer chains, of individual nano- or microparticles coated by such a polymer, and finally that of thin films made of such particles. Similar measurements are relevant when metallic conducting particles are used instead of polymer semiconducting ones. In fact, gold colloids have been recently used, e.g., to produce semitransparent conductive films $s^{7,8}$. Evaporation driven self assembly on a prestructured polymer or glass surface causes the arrangement of the colloids along well defined straight lines, forming a regular network. The conductivity of such thin films made from conductive metal or latex particles is usually characterized by macroscopic methods, like by the linear four point probe technique ${ }^{9,10}$, by the van der Pauw method ${ }^{11}$, or by impedance spectroscopy ${ }^{12}$. Very few studies have concentrated on the characterization of the conductivity of nano-, or microparticles ${ }^{13-16}$. In most works, particles were connected to two point nanoelectrodes and the current was measured upon applying a DC voltage between the electrodes. The small contact area (from a few nanometers to several micrometers square) between the electrodes and the conductive particles could result in non-negligible contact resistances, which affect considerably the measured conductivities.

In this paper, we introduce a direct method to measure the conductivity of individual particles by a microscopic four-point probe ( $\mu-4 \mathrm{PP})$ method. The four-point probe geometry allows for minimizing contact resistances between electrodes and particles. By using the focused ion beam (FIB) technique, we were able to fabricate nanoleads between four microelectrodes on a silicon chip and an individual particle, and to measure the particle's conductivity via a sensitive source meter. The distance $d$ between two adjacent microelectrodes was kept equal in order to apply the concept of the macroscopic four-point probe method ${ }^{9,10}$. Up to sixteen particles can be taken up by each chip, which allows for multiple conductivity measurements by simply multiplexing the electric contacts connected to the source meter (Figure 1). Although, for demonstration, we used full Au (conducting) and 

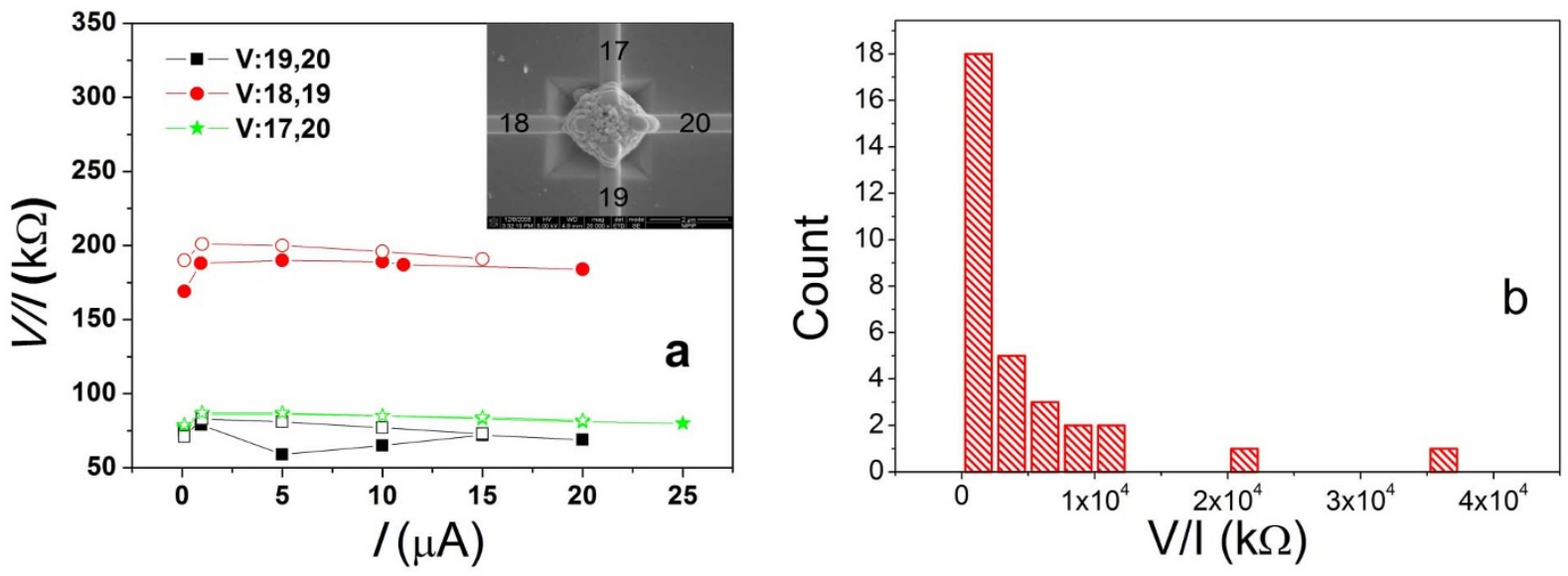

Figure $1 \mid$ (a) V/I of a Ag core-shell particle upon increasing (full symbols) and decreasing (hollow symbols) I; inset: SEM image of the Ag core-shell particle connected with microelectrodes; (b) histogram of V/I values for 23 particles.

Ag-coated latex particles (semiconducting) of a few micrometers in diameter, the method can be applied to other types of conducting or semiconducting particles of different diameters.

\section{Results}

Conductivity measurement of Ag core-shell particles. We first measure the conductivity of Ag core-shell particles using micro four point probe method to prove the ohmic contact. Figure 1(a) shows a representative plot of $V / I$ for a Ag core-shell particle upon applying different values of $I$. Squares and circles represent the voltage recorded upon respectively ramping up and down the current. Each curve represents a different connection of the electrodes with the voltage meter, as indicated in the inset. The measured values of $V /$ $I$ are similar (80-200 k $\Omega$ ) for all three connections. For each connection $V / I$ is independent on $I$, as shown by the nearly horizontal plots.

Statistic analysis was carried out on conductivity measurement of Ag core-shell particles to further prove the feasibility of the method. Figure 1(b) represents a histogram of $V / I$ values of 23 particles from two chips. The values of $V / I$ of most particles lie in the range from $100 \mathrm{k} \Omega$ to $10 \mathrm{M} \Omega$. The main reasons for the discrepancy come (i) from the reproducibility of the deposition process of the Pt connections between the Ti-Au microelectrode and the spherical Ag particle; and especially (ii) from inhomogeneities of the Ag shell of different particles. We chose $V / I=100 \mathrm{k} \Omega$ for the calculations. The conductivity $\sigma$ of a Ag core-shell particle of diameter $d$ and shell thickness $t$ is calculated by a simplified equation valid for planar surfaces and electrodes arranged in a square geometry (see Supporting Information).

$$
\sigma=\frac{0.11}{t(V / I)}, \text { valid for } t \ll a
$$

$a$ is the distance between the two adjacent microelectrodes. Since we are interested in an estimate of the order of magnitude of the conductivity, Eq. (1) is a good approximation also for a spherical geometry. According to values obtained from SEM images (Fig. S4a), $t \approx$ $200 \mathrm{~nm}$ and $a \approx 1200 \mathrm{~nm}$. For $V / I=100 \mathrm{k} \Omega$, the conductivity results $\sigma \approx 0.055 \mathrm{~S} / \mathrm{cm}$.

The content of impurity, i.e. S inside Ag layer of the particles could influence the conductivity of the layer, and thus the entire particle. Hence elemental analysis was carried out on Ag core-shell particles. Figure 2 displays the atomic ratio of sulfur to silver (S:Ag) in $10 \mathrm{Ag}$ core-shell particles, as measured by energy dissipation X-ray spectroscopy (EDX). According to the composition of the core-shell particle, the absorption signal of $S$ in the EDX spectra only results from $\mathrm{Ag}_{2} \mathrm{~S}$. The average $\mathrm{S}: \mathrm{Ag}$ ratio is $\sim 0.3$, indicating that almost $50 \%$ of $\mathrm{Ag}$ was converted into $\mathrm{Ag}_{2} \mathrm{~S}$.
Conductivity measurement of all-Au particles. We directly deposited $\mathrm{Pt}$ nanoleads between the $\mathrm{Au}$ particle and the four microelectrodes used for the bottom contact as explained in method section (Figure $3 a$ ). Figure $3 b$ shows $V / I$ values of a single $\mathrm{Au}$ particle upon applying different currents $I$. Each curve represents the corresponding electrodes combination (as indicated by the numbers) connected to the voltmeter. $V / I \approx 100 \Omega$ and is nearly constant over the whole range.

Conductivity measurement of FIB deposited Pt electrodes. The Pt electrodes deposited by FIB are used to connect particles and microelectrodes, as shown previously. To measure the conductivity of the particles, contact between the Pt connections and the $\mathrm{Ti}-\mathrm{Au}$ microelectrodes needs to be ohmic. We therefore measure the resistance $R$ between two $\mathrm{Ti}-\mathrm{Au}$ electrodes connected by $\mathrm{Pt}$ connections deposited by FIB (figures 4 and S3). For seven tested connections we found $R \approx 125 \pm 40 \Omega$. We calculated the resistivity $\rho$ of the FIB deposited Pt with $\rho=R \frac{A}{l}$, where $A$ is the cross-sectional area and $l$ is the length of the resistor. We calculated $\rho \approx 2.4 \times 10^{-3} \Omega$ $\mathrm{cm}$, with $l=700 \mathrm{~nm}$ and $A=700 \times 200=1.4 \times 10^{5} \mathrm{~nm}^{2}$, which is 2-3 orders of magnitude higher than that of pure Pt $\left(\rho=1.04 \times 10^{-5}\right.$ $\Omega \mathrm{cm})^{18}$. This result is in good agreement with literature ${ }^{17}$ since the deposited Pt electrode contained around 50\% carbon. The deposited $\mathrm{Pt}$ connections are thus in ohmic contact with the $\mathrm{Ti}-\mathrm{Au}$ microelectrodes, and this validates the conductivity measurements of individual conductive particles by top contact electrodes.

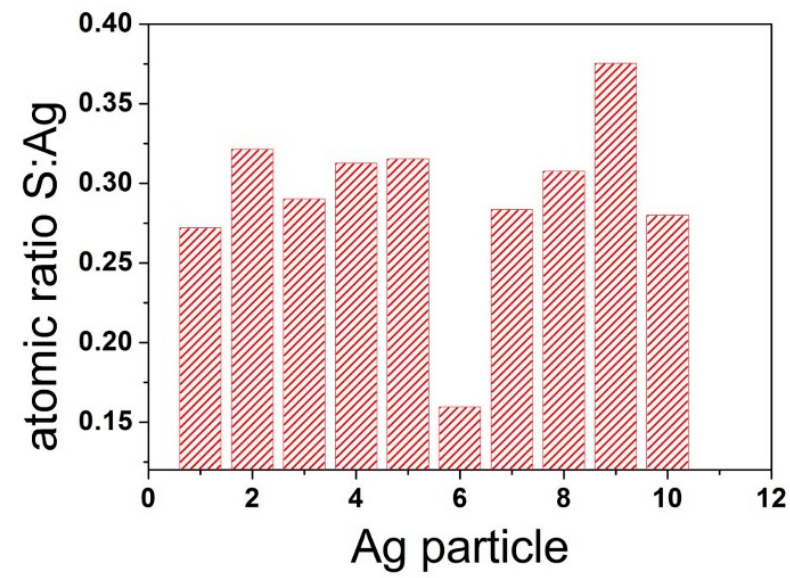

Figure $2 \mid$ Atomic ratio of S:Ag in $10 \mathrm{Ag}$ core-shell particles, as measured by EDX spectroscopy. 

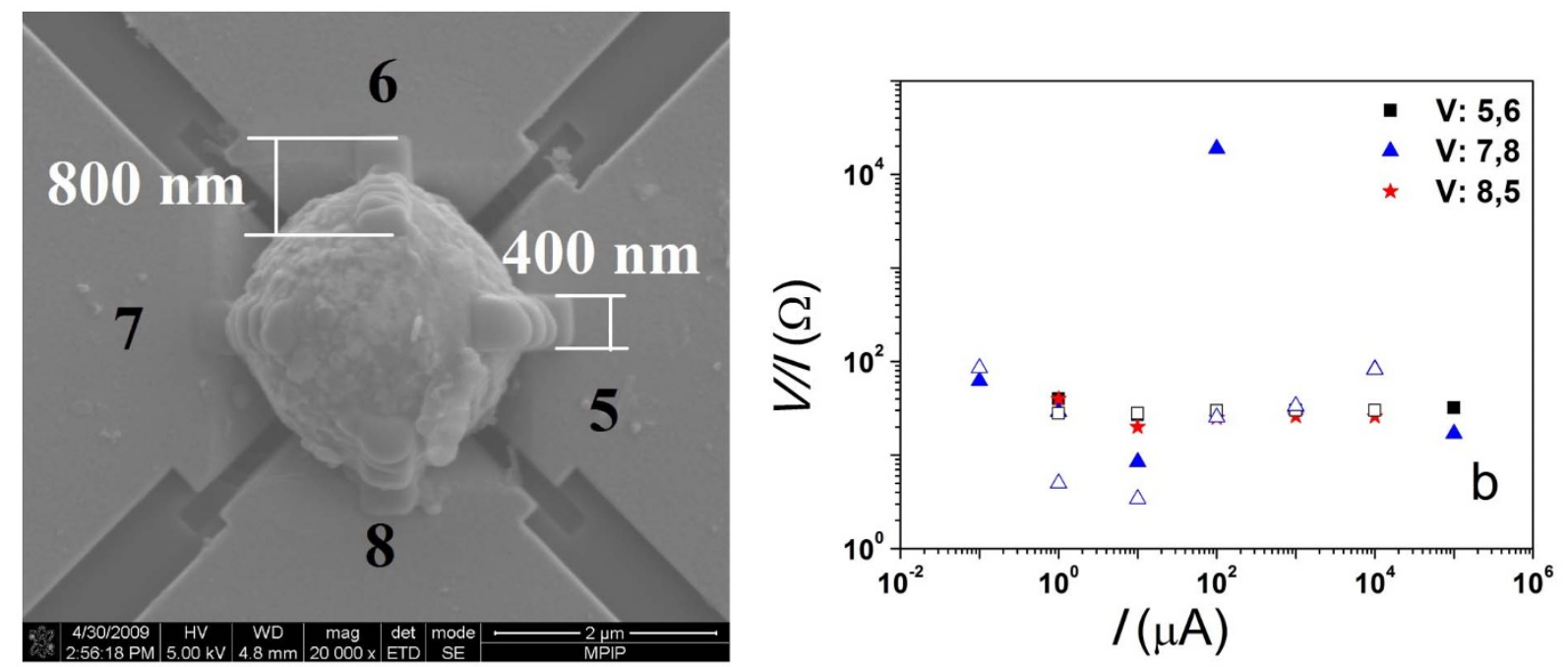

Figure 3 (a) SEM image of an all-Au particle connected by four Pt electrodes; (b) plot of V/I measured upon increasing (full symbols) and decreasing $I$ (hollow symbols). (color online)

\section{Discussion}

The distribution of the electric field inside the conductive layer of the core-shell particles depends on thickness and conductivity. Therefore, the measured resistance $(R=V / I)$ and the equations for calculating the conductivity are different for core-shell and full-metal particles. We discuss the results on Ag core-shell particles and all-Au particles separately.

The V/I values of Ag core-shell particles are similar, indicating that also the contact areas are similar. For metals $V / I$ is constant, according to Ohm's law. For polymers (semi-conducting or insulating), on the other hand, $I$ is proportional to $V^{2}$, as described by the MottGurney equation $^{19}$. The constant value of $V / I$ over the whole measuring range (Figure 1) thus indicates that the current flows in the conductive Ag shell, i.e. through percolation channels formed by the nanoscopic conductive Ag domains.

The calculated conductivity of the Ag core-shell particle (ca. $0.055 \mathrm{~S} / \mathrm{cm}$ ) is much lower than that of pure $\mathrm{Ag}$, i.e. $6.3 \times 10^{5} \mathrm{~S} /$ $\mathrm{cm}^{20}$. There could be several reasons for this. Firstly, the conductive Ag layer is only $\sim 200 \mathrm{~nm}$ thick and not homogeneous, while the bulk is made of insulating melamine resin. The current "percolates" between neighboring Ag domains, leading to a lower conductivity of the shell. Secondly, Ag reacts with sulfur compounds in the atmosphere, and thus can form $\mathrm{Ag}_{2} \mathrm{~S}$ when stored in air for some time. The

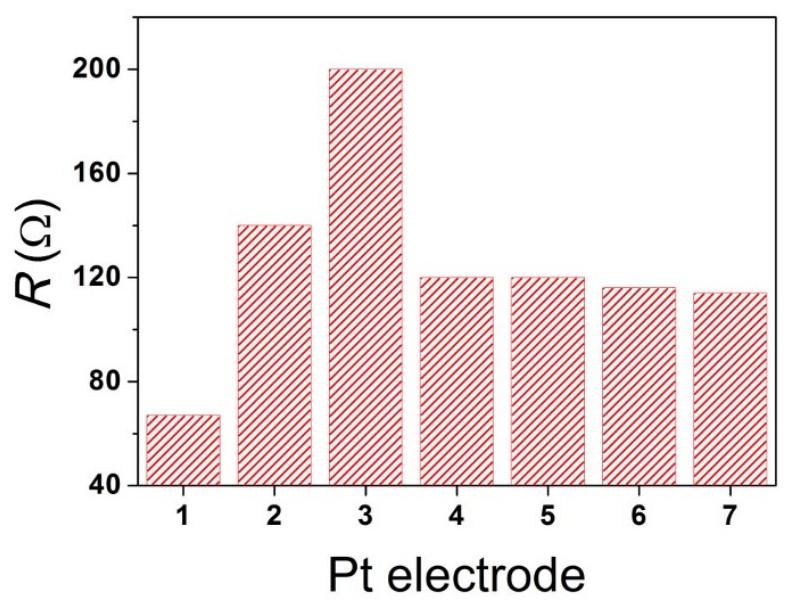

Figure $4 \mid$ Resistance $R$ between the Ti-Au electrodes of the array and the Pt electrodes deposited by FIB. conductivity of $\mathrm{Ag}_{2} \mathrm{~S}$ is $10^{-3}-10^{-5} \mathrm{~S} / \mathrm{cm}^{21-23}$, which is much lower than Ag. According to the calculation, almost $50 \%$ of Ag was converted into $\mathrm{Ag}_{2} \mathrm{~S}$, we therefore attribute to this process the main reason for the low conductivity of the Ag core-shell particles.

We started conductivity measurements of Au bulk particles using the bottom-contact geometry (see methods section: fabrication of nanocontacts). Upon placing the Au particle inside the pyramidal pit the four electrodes should be connected by the Au particle, and the measured resistance V/I should decrease. However, similar as with the control measurements with the Ag particles (see supporting information), $V / I$ of all tested Au particles were also in the range of $\mathrm{T} \Omega$. This is too high for conductive full metal particles. In the bottom contact geometry, the contact area between the particles and the $\mathrm{Ti}$ Au microelectrodes is too small (ideally a point contact) to guarantee a constant measurable current. In addition, the particles did not sit in the very center of the pit (Figure S5), which results in four different contact areas. Such issues make the "bottom contact" geometry unsuitable for four-point probe measurements, where the four contact areas must be equal. Whereas for top-contact geometry, the four curves (Figure 3b) almost overlap, with few exceptions. Such results prove that the top-contact geometry is suitable for conductivity measurements of individual particles. The diameter of the all-Au particles is between $1.5-3 \mu \mathrm{m}$, which is of similar length scale as $d$ $(\sim 1.6 \mu \mathrm{m}$, Figure S5b). Equation (1) is thus not applicable to $\mathrm{Au}$ particles.

In summary, we have demonstrated a method for measuring hybrid metal, semiconducting core-shell and full-metal conducting particles by a microscopic four-point probe ( $\mu-4 \mathrm{PP})$ method. By using a focused ion beam, we were able to connect individual particles with microelectrodes via Pt nanoleads and to measure the $I-V$ curves by a high precision source meter. To test the applicability of the method, we used all-Au and Ag-polymer core-shell particles as models. The measured conductivities were comparable to the expected conductivities of the two particles. The deviation of the two values (measured and theoretical) can be attributed to geometrical effects. Although in this study we only showed examples of Au and Ag particles with a diameter of $\sim 2 \mu \mathrm{m}$, this method could easily be extended to other particles of different sizes in the micrometer or sub-micrometer range.

\section{Methods}

Full metal and hybrid metal/polymer particles. Hybrid Ag core-shell particles. Agcoated melamine resin particles (diameter $\sim 2 \mu \mathrm{m}$, figure $\mathrm{S1}(\mathrm{a})$ ) were purchased from Microparticles GmbH (Berlin, Germany). The thickness of the Ag shell around 


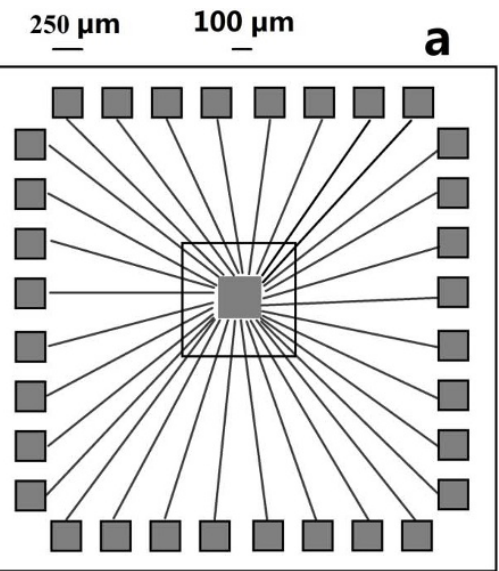

b

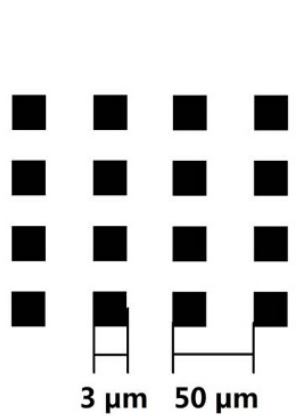

C

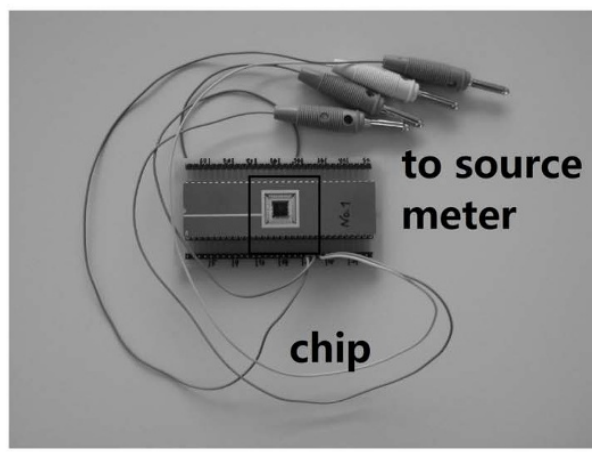

Figure $5 \mid$ Schematics of a silicon chip (a) and the center area of it (b); (c) photo of a wired chip ready for micro four-point-probe ( $\mu$-4PP) measurements.

the particle core was determined according to the cross-section images of the particles measured by scanning electron microscope (SEM, FEI Nova 600 Nanolab, Eindhoven, The Netherlands). The cross-section was generated by focused ion beam milling (FIB, FEI Nova 600 Nanolab). The thickness of the Ag layer was 100-200 nm (figure S1(b)). This value was used to calculate the conductivity of the Ag core-shell particles.

Full metal Au particles. The all-Au particles (diameter 1.5-3.0 $\mu \mathrm{m}$, figure $\mathrm{S1}(\mathbf{c})$ ) were purchased from Johnson Matthey GmbH (Karlsruhe, Germany). The actual size of individual Au particle was determined by SEM. Some Au particles were also milled by FIB. The SEM image after the FIB milling confirmed that the particles entirely consisted of $\mathrm{Au}$ (figure S1d)

Design of the electrode arrays. Figure 5(a) is a schematic sketch of silicon chips we used to perform $\mu$-4PP measurements on individual particles. There are 16 pyramidal pits etched on each silicon chip. The dimension of each pit is $3 \times 3 \mu \mathrm{m}^{2}$ and the distance between two neighboring pits is $50 \mu \mathrm{m}$ (figure $\mathbf{5}(\mathbf{b})$ ). This dimension is designed for particles with a diameter of 1-3 $\mu \mathrm{m}$, but is also scalable for smaller or larger sizes. Each silicon chip is also covered by a layer of $200 \mathrm{~nm} \mathrm{SiO} 2$ for electric insulation. The pre-evaporated microelectrodes $(10 \mathrm{~nm}$ Ti and $200 \mathrm{~nm} \mathrm{Au})$ were deposited by thermal evaporation. Each electrode was electrically bonded to a socket, which is connected to a source meter (figure 5(c)). As the used chip can be detached from the socket, the socket is reusable.

Fabrication of the nano contacts. Particles were first suspended into water. A drop of the suspension was then deposited on the array (figure 6(a)), and blew off by an air gun gently to remove excess liquid. After evaporation of the liquid, some particles were left behind. Each particle was then moved inside a pyramidal pit by a tipless silicon nitride cantilever (NP-O, Veeco Instrument Inc., Santa Barbara, CA) controlled by a hydraulic micromanipulator (MMO-203, Narishige, Ltd., Tokyo, Japan). All 16 pits were filled with a particle. The surfaces of pyramidal pits are bare, and the four microelectrodes are $3 \mu \mathrm{m}$ away from the edge of the pyramidal pit (figure 6(b)). To establish a nanocontact between an individual particle and the microelectrodes, Pt nanoelectrodes (with $\sim 50 \%$ carbon $^{18}$ ) were deposited by FIB, connecting each particle to the four Ti-Au electrodes (figure 6(c)). The thickness of the Pt electrode was around $200 \mathrm{~nm}$. The dimension of each deposited Pt electrode was $4800 \times 400 \mathrm{~nm}^{2}$ (length $\times$ width) for Ag core-shell particles, and $800 \times 400 \mathrm{~nm}^{2}$ (length $X$ width) for all-Au particles. The position of each Pt nanoelectrode was carefully controlled by SEM inspection to achieve four similar contact areas between the electrode and the particle of around $400 \times 400 \mathrm{~nm}^{2}$. As the particle was connected to the electrodes from the top; we called this geometry "top contact". We also designed another geometry for control experiments, where each pyramidal pit was pre-coated with a layer of Ti-Au and connected to the four surrounding microelectrodes (figure S2). The particles were connected to the microelectrodes at the bottom; we called it "bottom contact" geometry. The detailed fabrication procedure can be found in supporting information.

Microscopic four-point probe measurement ( $\mu$-4PP). A source meter (Mod. 6430, Keithley Instruments GmbH, Germering, Germany) was used to measure the conductivity of individual particles. The connection between the four probes of the source meter and the microelectrodes was established via a socket, as described previously (Figure 5c). Every two adjacent electrodes were connected to the voltmeter, and the other two to the current source (constant current) of the source meter. For each particle connected by four electrodes there were four different connections to the source meter, corresponding to the van der Pauw electrode geometry ${ }^{11}$. For each connection, constant $I$ was applied and $V$ was measured. I was also ramped up and down, and $V$ was measured for each of $I$ respectively.

EDX measurement on Ag core-shell particles. Energy dissipation X-ray analysis (EDX, FEI Nova 600, Nanolab) was performed on Ag core-shell particles to measure the content of sulfur in the conducting Ag shell of the particles. The atomic ratio of sulfur to silver was analyzed from the resulting spectra.

Pt electrodes for contact resistance test. To check the contact resistance between the deposited Pt electrodes and the Ti-Au electrodes, a single Pt wire was deposited by FIB to connect two adjacent Ti-Au electrodes (figure S3). The dimension of the Pt electrodes was controlled to be $2100 \times 700 \times 200 \mathrm{~nm}^{3}$ (length $\times$ width $\times$ height). The resistance between the two connected $\mathrm{Ti}$-Au electrodes was then measured with the source meter.

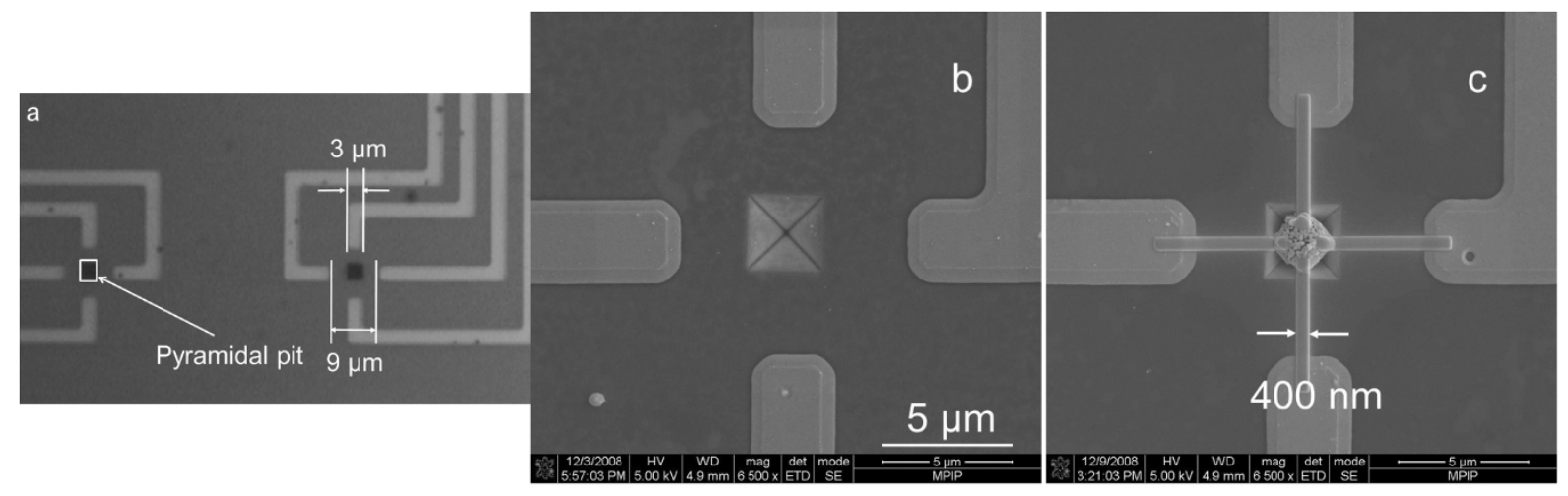

Figure $6 \mid$ (a) Optical microscopy image of electrode arrays (magnification 50×); (b) SEM image of top contact design; (c) SEM image of four electrodes connected to a Ag core-shell particle by Pt electrodes deposited by FIB. 
1. Singh, T. B. \& Sariciftci, N. S. Progress in plastic electronics devices. Annual Review of Materials Research 36, 199-230 (2006).

2. Kus, M. \& Okur, S. Electrical characterization of PEDOT:PSS beyond humidity saturation. Sensors and Actuators B-Chemical 143, 177-181 (2009).

3. Moons, E. Conjugated polymer blends: linking film morphology to performance of light emitting diodes and photodiodes. Journal of Physics-Condensed Matter 14, 12235-12260 (2002).

4. Li, Y. F. \& Hou, J. H. Major Classes of Conjugated Polymers and Synthetic strategies (CRC Press Inc., Boca Raton, 2008).

5. Wang, J. J. et al. Facile Synthesis of Spherical Polyelectrolyte Brushes as Carriers for Conducting Polymers to be Used in Plastic Electronics. Macromolecular Chemistry and Physics 210, 1504-1509 (2009)

6. Wang, J. J. et al. Construction of Redispersible Polypyrrole Core-Shell Nanoparticles for Application in Polymer Electronics. Advanced Materials 21, 1137-1141 (2009).

7. Higashitani, K., McNamee, C. E. \& Nakayama, M. Formation of Large-Scale Flexible Transparent Conductive Films Using Evaporative Migration Characteristics of Au Nanoparticles. Langmuir 27, 2080-2083 (2011).

8. Vakarelski, I. U., Chan, D. Y. C., Nonoguchi, T., Shinto, H. \& Higashitani, K. Assembly of Gold Nanoparticles into Microwire Networks Induced by Drying Liquid Bridges. Physical Review Letters 102, (2009).

9. Valdes, L. B. Resistivity Measurements on Germanium for Transistors. Proceedings of the Institute of Radio Engineers 42, 420-427 (1954).

10. Smits, F. M. Measurement of Sheet Resistivities with the 4-point Probe. Bell System Technical Journal 37, 711-718 (1958)

11. van der Pauw, L. J. A method of measuring specific resistivity and Hall effect of discs of arbitrary shape. Philips Research Reports 13, 1-9 (1958).

12. Mpoukouvalas, K., Wang, J. J. \& Wegner, G. Conductivity of Poly(pyrrole)Poly(styrene sulfonate) Core-Shell Nanoparticles. Chemphyschem 11, 139-148 (2010).

13. Cabrini, S. et al. Cross beam lithography (FIB plus EBL) and dip pen nanolithography for nanoparticle conductivity measurements. J. Vac. Sci. Technol. B 23, 2806-2810 (2005).

14. Gover, L. B., Ludtke, G. H., Haug, T. \& Parisi, R. J. J. Charge transport through a single particle located in between nanogap electrodes. Physics Letter A $\mathbf{3 7 4}$ 3328-3331 (2010)

15. Sun, T. B. \& Morgan, C. H. Single-Colloidal Particle Impedance Spectroscopy: Complete Equivalent Circuit Analysis of Polyelectrolyte Microcapsules. Langmuir 26, 3821-3828 (2010).

16. Dayen, J. F. et al. Nanotrench for nano and microparticle electrical interconnects. Nanotechnology 21, 335303-335310 (2010).

17. Platinum Deposition Technical Note, PN 403527221851-B, (FEI Company, Oregon, 2003).
18. Barbalace, K. Periodic table of elements - Platinum, EnvironmentalChemistry.com (1995-2013), Accessed on-line: 5/12/2013, http://EnvironmentalChemistry.com/ yogi/periodic/Pt.html.

19. Schwoerer, M. \& Wolf, H. C. Organic Molecular Solids (Willey-VCH, Weinheim, 2007).

20. Barbalace, K. Periodic table of elements - Silver, EnvironmentalChemistry.com (1995-2013), accessed on-line: 5/12/2013, http://EnvironmentalChemistry.com/ yogi/periodic/Ag.html.

21. Dhumure, S. S. \& Lokhande, C. D. Solution growth of silver sulfide thin-films. Materials Chemistry and Physics 27, 321-324 (1991).

22. Grozdanov, I. Solution growth and characterization of silver sulfide films. Applied Surface Science 84, 325-329 (1995).

23. El-Nahass, M. M., Farag, A. A. M., Ibrahim, E. M. \& Abd-El-Rahman, S. Structural, optical and electrical properties of thermally evaporated Ag2S thin films. Vacuum 72, 453-460 (2004).

\section{Acknowledgements}

We thank Ciba S.C. (part of BASF) and The Max Planck Society (MPG) for financial support. We also thank Dirk Richter for help with the electronics, Michael Kappl and Maren Müller for introducing the FIB, Cheng Wei and Andreas Best for support during the design and testing phase of the $\mu-4 \mathrm{PP}$ setup, Denis Andrienko for support with the conductivity model, and Hans-Jürgen Butt and Gerhard Wegner for fruitful discussions.

\section{Author contributions}

L.S. wrote the main manuscript text and prepared the figures. J.J.W. and E.B. supervised the project, and revised the manuscript. All authors reviewed the manuscript.

\section{Additional information}

Supplementary information accompanies this paper at http://www.nature.com/ scientificreports

Competing financial interests: The authors declare no competing financial interests.

How to cite this article: Sun, L., Wang, J. \& Bonaccurso, E. Conductivity of individual particles measured by a microscopic four-point-probe method. Sci. Rep. 3, 1991; DOI:10.1038/srep01991 (2013).

(c) (i) (-) $\odot$ This work is licensed under a Creative Commons Attribution-

NonCommercial-NoDerivs Works 3.0 Unported license. To view a copy of this license, visit http://creativecommons.org/licenses/by-nc-nd/3.0 